\title{
DAY 1 DIAGNOSIS OF DENGUE FEVER
}

Shyam P1 , Sreelatha P2 , Saswati Chattopadhyay³, Raja Mukherjee ${ }^{4}$, Tarkeshwar Yadav 5

\section{HOW TO CITE THIS ARTICLE:}

Shyam P, Sreelatha P, Saswati Chattopadhyay, Raja Mukherjee, Tarkeshwar Yadav. "Day 1 Diagnosis of Dengue Fever". Journal of Evolution of Medical and Dental Sciences 2014; Vol. 3, Issue 44, September 15;

Page: 10915-10919, DOI: $10.14260 /$ jemds/2014/3425

ABSTRACT: BACKGROUND: Dengue is an RNA virus of the family Flaviviridae transmitted by Aedes mosquitoes particularly Aedes aegypti. It is widely distributed throughout the tropics and subtropics and in a small proportion of cases the virus leads to life threatening complications dengue hemorrhagic fever and dengue shock syndrome. OBJECTIVES: To study the early diagnosis of Dengue on day 1 as there is no vaccine or specific antiviral treatment available. METHODS: A prospective study of 104 patients was done based on clinical criteria of Dengue. RESULTS: Out of 104 serum samples 46 (44\%) were positive by NSI Ag MICROELISA, 37 (35\%) by NSI antigen IMMUNO CHROMATOGRAPHY. 3 (2\%) samples are positive by IgM IMMUNO CHROMATOGRAPHY and only one sample was positive for IgG IMMUNOCHROMATOGRAPHY. CONCLUSION: The present study has established the significance of NSI Ag MICROELISA with NSI antigen IMMUNO CHROMATOGRAPHY in increasing the diagnostic efficiency in the day 1 diagnosis of Dengue fever.

KEYWORDS: Dengue NS1 Ag MICROELISA, NS1 antigen Immuno chromatography, IgM immuno chromatography, IgG immunochromatography.

INTRODUCTION: Dengue fever or break bone fever is a tropical disease caused by a RNA virus of the family Flaviviridae genus Flavivirus. It is primarily transmitted by Aedes aegypti mosquitoes. These mosquitoes bite primarily during the daytime and humans are the primary host. An infection can be acquired by a single bite of the mosquitoes. It is endemic in more than 110 countries infecting about 50-100 million people each year ${ }^{1}$ worldwide with 500, 000 cases of dengue hemorrhagic fever (DHF) and dengue shock syndrome (DSS) and around 30, 000 deaths, mostly amongst children. ${ }^{2}$

The incidence has increased 30 folds between 1960 and $2010^{12}$ probably due to a combination of urbanization, population growth, increased international travel and global warming. ${ }^{1}$

Usually the infection is asymptomatic (80\%) and only in a small proportion is it life threatening. The incubation period range from 3-14 days but most often it is 4-7 days. The characteristic symptoms are sudden onset high fever, headache, retro-orbital pain, muscle and joint pains and a rash.

Early diagnosis plays a significant role in the treatment, control of an impending epidemic and vector control measures. The major diagnostic methods currently available are viral culture, viral RNA detection by reverse transcriptase PCR (RT-PCR) and serological tests such as an immunoglobulin M (IgM) capture enzyme-linked immunosorbent assay (MAC-ELISA). However, early diagnosis still remains a problem, as all these assays have their own pitfalls.

The first two assays have restricted scope as a routine diagnostic procedure. Viral isolation by cell culture and subsequent detection by immunofluorescence, though the gold standard, ${ }^{3}$ cannot be used as a routine diagnostic procedure due to its low sensitivity, laborious procedure and time consumption. ${ }^{4,5}$ 
The requirement of a highly trained staff, the need of sophisticated equipment as well as the cost factor associated with molecular methods has limited its application as a routine diagnostic assay. The MAC-ELISA, which is a commonly used assay, has a low sensitivity in the first four days of illness. ${ }^{6}$ The requirement of paired sera at acute and convalescent phase, which improves the accuracy of the diagnosis, further delays it.

The viral genome contains about 11, 000 nucleotide bases and several other protein molecules found only in infected host cells required for the replication of the virus. Among the nonstructural protein NS1 (non-structural protein 1) is a highly conserved glycoprotein that is essential for the viability of the virus and is produced both its membrane-associated and secretary forms. ${ }^{7}$

Enzyme-linked immunosorbent assays (ELISA) directed against NS1 antigen (NS1 Ag) have demonstrated its presence at high concentrations in the sera of Dengue infected patients during the early clinical phase of the disease. ${ }^{7}$ The detection of secretary NS1 protein represents a new approach to the diagnosis of acute infection.

The study aims at early diagnosis of Dengue i.e. on day 1 by NSI Ag MICROELISA and NSI antigen IMMUNO CHROMATOGRAPHY taking the ELISA as the gold standard as it is a very sensitive test.

MATERIALS AND METHODS: The study was carried out in patients attending a tertiary care hospital in South India from July to December 2011. In this community based cross - sectional study, a total of 104 patients presenting with high fever, retro orbital pain, muscle and joint pains, flushed skin, macula-papular rash, petechiae, nausea, vomiting with/without bleeding manifestations in both sexes and all age groups were selected for the study. They were clinically suspected to be suffering from dengue virus infection. The patients attended the Medicine OPD for treatment and were send to the Microbiology Dept for confirmation of the diagnosis.

Detailed history was taken and clinical examination was carried out in all the patients. $5 \mathrm{ml}$ of venous blood was drawn aseptically from each of them. The serum was separated from the blood samples by centrifugation at $1500 \mathrm{rpm}$ for 15 minutes and was stored at $-20{ }^{\circ} \mathrm{C}$. Investigations were performed to exclude Chikungunya, malaria, leptospirosis, typhoid fever and meningococcal disease. ${ }^{8}$

RESULTS: All 104 serum samples were tested by dengue NSI Ag MICROLISA as well as Immunochromatography for the detection of NSI antigen and IgM, IgG antibodies and all the results were compared.

Of the 104 clinically suspected dengue cases, 46 patients were positive by NS1 Ag MICROELISA and of these 46 samples 37 samples were also positive by dengue NS1 ICT positive Hence the diagnosis of Dengue was correlated in 37 cases by both methods. NS1 ICT failed to identify in 9 cases.

Three isolates were positive by dengue IgM ICT. Only one sample was positive for dengue IgG antibody and also for IgM ICT as shown in the table I.

High unremitting fever was the commonest symptom seen in all the 46 confirmed cases together with flushed skin, headache and muscle pains. Retro-orbital pain was present in 40 seropositive cases.

Joint pain was present in 21 sero-positive cases. The patients gave history that the pain started on the first or second day of fever. It then involved the spine, both the small and big joints of 
both the extremities with no upper/lower limb predilection. Radiological screening of the joints did not reveal any significant abnormality.

Rash with fever was present in 17 out of 46 positive cases. The rashes were generalized, erythematous, maculopapular in nature in both the trunk and extremities. Aphthous ulcers were also found to be present on the oral mucosa and the tongue.

Hemorrhage as petechiae and bleeding manifestations' was present only in 1 sero-positive cases as shown in Table 2.

Anorexia, nausea and pain abdomen were the other associated minor symptoms. Nausea and vomiting was present in 37 cases while anorexia was complained by all 104 patients. Abdominal muscle ache together with other muscle ache was complained by 46 patients-Table 2 .

All the patients had normal blood counts except for one case who presented with high fever, muscle and joint pains, severe headache, petechiae and epistaxis, conjunctival ecchymosis and bleeding per vagina.

The patient was positive for NSI Ag in MICROELISA and ICT for NSI antigen. Later on the patient was also positive in ICT for IgM and IgG antibodies. The patient however died due to uncontrollable bleeding per vagina after 2 weeks in spite of adequate treatment. Rest of the patients was treated symptomatically and all recovered within 1 to 3 weeks.

DISCUSSION: The diagnosis of dengue is clinically made on the basis of the symptoms and physical examination findings. The morbidity and the mortality of DHF can be reduced by early diagnosis, hospitalization and symptomatic care to combat the fatal consequences of it like dengue hemorrhagic fever and shock.

NS1 Ag circulates uniformly in all serotypes of the virus and it circulates at high level during the first few days of illness. ${ }^{9} \mathrm{NS} 1 \mathrm{Ag}$ levels varies from $0.04-2 \mu \mathrm{g} / \mathrm{ml}$ in acute-phase serum samples, to only $0.04 \mu \mathrm{g} / \mathrm{ml}$ or even less in convalescent phase serum. ${ }^{6}$ This is the reason for its higher detection rate in acute phase sera.

NS1 Ag MICROELISA and dengue NS1 ICT gives results on Day 1 of the fever. Hence commercial kits of dengue NS1 antigen are useful for early laboratory diagnosis but need to be confirmed by NS1 Ag MICROELISA. ELISA has been used as the gold standard for DENV infection detection.

There was 104 clinically suspected dengue cases, of these 46 patients tested positive by NS1 Ag MICROELISA and dengue NS1 ICT could correlate only in 37 samples already tested positive by NS1 Ag MICROELISA. Hence Dengue NSI Ag MICROELISA is a more sensitive method than dengue NS1 ICT leading to a difference in the results between 2 tests.

ICT for IgM showed low positivity as it takes 3 to 5 days for IgM antibodies and anti-DENV for IgG takes 10-14 days to become detectable. Hence of Dengue NS1 Ag MICROELISA assisted by dengue NS1 ICT plays a very important role for day 1 diagnosis of the fever.

CONCLUSION: NSI antigen is an effective tool for diagnosis of Dengue infection, especially within the first four days of illness. Early detection of DHF by NS1 assay can help in early confirmation and management of this vulnerable group. 
NS1 Ag assay, if used in combination with MAC-ELISA on a single serum sample of a suspected case, has the ability to improve the diagnostic algorithm contributing significantly to the clinical treatment and control of dengue viral infections.

\section{REFERENCES:}

1. Smith AW, Chen LH, Massad E, Wilson ME. Threat of dengue to blood safety in dengue-endemic countries. Emerg Infect Dis 2009; 15:8-11.

2. Guzman MG, Kouri G. Dengue: an update. Lancet Infect Dis 2002; 2: 33-42.

3. Chakravarti A, Kumaria R, Batra VV, Verma V. Improved detection of dengue virus serotypes from serum samples - Evaluation of single-tube multiplex RT-PCR with cell culture. Dengue Bulletin 2006; 30: 133-40.

4. Sekaran SD, Lan EC, Mahesawarappa KB, Appanna R, Subramanium G. Evaluation of a dengue NS1 capture ELISA assay for the rapid detection of dengue. J Infect Developing Countries 2007; 1:182-8.

5. Shu PY, Huang JH. Current advances in dengue diagnosis. Clinical and Diagnostic Laboratory Immunology 2004; 11:642-50.

6. Alcon S, Talarmin A, Debruyne M, Falconar A, Duebel V, Flamand M. Enzyme-Linked Immunosorbent Assay Specific to Dengue Virus Type 1 Nonstructural Protein NS1 Reveals Circulation of the Antigen in the Blood during the Acute Phase of Disease in Patients Experiencing Primary or Secondary Infections. J Clin Microbiol 2002; 40:376-81.

7. Dussart P, Labeau B, Lagathu G, Louis P, Nunes MRT, Rodrigues SG, et al. Evaluation of an Enzyme Immunoassay for detection of dengue virus NS1 antigen I human serum. Clin Vaccine Immunol 2006; 13: 1185-9.

8. Kumarasamy V, Wahab AHA, Chua SK, Hassan Z, Chem YK, Mohamed M, Chua KB. Evaluation of a commercial dengue NS1 antigen-capture ELISA for laboratory diagnosis of acute dengue virus infection. J Virol Methods 2007; 140: 75-9.

9. Bessof K, Delorey M, Sun W, Hunsperger E. Comparison of Two Commercially Available Dengue Virus (DENV) NS1 Capture Enzyme-Linked Immunosorbent Assays Using a Single Clinical Sample for Diagnosis of Acute DENV Infection. Clin Vaccine Immunol 2008; 15: 1513-8.

\begin{tabular}{|l|c|}
\hline \multicolumn{1}{|c|}{ Diagnostic test } & No. of positive serum samples \\
\hline Dengue NSI ELISA & 46 \\
\hline Dengue NSI ICT. & 37 \\
\hline Dengue IgM ICT.. & 3 \\
\hline Dengue IgG ICT.$\cdots$ & 1 \\
\hline
\end{tabular}

TABLE I: DENGUE POSITIVE SAMPLES BY DIFFERENT ASSAYS 
ORIGINAL ARTICLE

\begin{tabular}{|l|c|c|}
\hline \multicolumn{1}{|c|}{ Clinical criteria } & No. of cases & Percentage proportion \\
\hline Fever & 46 & $100 \%$ \\
\hline Headache & 46 & $100 \%$ \\
\hline Flushed Skin & 46 & $100 \%$ \\
\hline Muscular Pains & 46 & $100 \%$ \\
\hline Retro-orbital Pain & 40 & $86 \%$ \\
\hline Nausea \& Vomiting & 35 & $76 \%$ \\
\hline Joint pains & 21 & $45 \%$ \\
\hline Maculo-papular rash & 17 & $36 \%$ \\
\hline Petechiae \& Bleeding manifestations & 01 & $2.1 \%$ \\
\hline \multicolumn{2}{|c|}{ TABLE 2: COMPARATIVE EVALUATION BASED ON CLINICAL CRITERIA }
\end{tabular}

\section{AUTHORS:}

1. Shyam P.

2. Sreelatha P.

3. Saswati Chattopadhyay

4. Raja Mukherjee

5. Tarkeshwar Yadav

\section{PARTICULARS OF CONTRIBUTORS:}

1. Professor, Department of Dermatology, Mamata General Hospital, Khammam, Andhra Pradesh.

2. Professor, Department of Micro Biology, Mamata General Hospital, Khammam, Andhra Pradesh.

3. Demonstrator, Department of Micro Biology, IPGME \& R, Kolkata.

4. Medical Officer, Department Orthopaedics, Durgapur SD Hospital, West Bengal.
5. M.Sc Student, Department of Micro Biology, Mamata General Hospital, Khammam, Andhra Pradesh.

\section{NAME ADDRESS EMAIL ID OF THE CORRESPONDING AUTHOR:}

Dr. Saswati Chattopadhyay,

1, Amarnath Road,

Uttarpara Hooghly,

West Bengal-712258.

Email: dr.saswatichattopadhyay@gmail.com

Date of Submission: 26/08/2014.

Date of Peer Review: 27/08/2014.

Date of Acceptance: 09/09/2014.

Date of Publishing: 15/09/2014. 